\title{
A Brief Report of the Epidemiology of Obesity in the Inflammatory Bowel Disease Population of Tayside, Scotland
}

\author{
Helen Steed ${ }^{a}$ Shaun Walsh ${ }^{b} \quad$ Nigel Reynolds ${ }^{c}$ \\ ${ }^{a}$ Gut Group, University of Dundee, \\ ${ }^{\mathrm{b}}$ Department of Pathology, National Health Service Tayside, \\ ${ }^{c}$ Department of Gastroenterology, Ninewells Hospital, Dundee, UK
}

\section{Key Words}

BMI · Epidemiology · Malnutrition · Obesity · Overweight

\section{Summary}

Aim: Obesity in inflammatory bowel disease (IBD), particularly Crohn's disease (CD), has previously been considered unusual (3\%). CD patients who are obese tend to have increased perianal complications and a higher level of disease activity on an annual basis. Obesity in Scotland has been documented to have increased over the last decade, and over half all men and women in Scotland are now considered to be overweight. This study aims to assess obesity prevalence in the IBD community in Tayside, Scotland. Methods: All IBD patients ( $n=1,269$ ) were considered for inclusion. Inclusion criteria required a weight measurement taken from the preceding 12 months and a height measurement within the last decade. 489 patients were included in the analysis. Results: $18 \%$ of the Tayside IBD population were obese in comparison to approximately $23 \%$ of the Scottish population on a whole. A further $38 \%$ of patients were overweight, the same percentage as the general population. In the overweight and obese ulcerative colitis patients there were higher levels of surgery, but the converse was true in the CD group, where the normal-weight group had the highest levels of surgery. There were significantly more obese men and women with CD than with ulcerative colitis $(P=0.05)$. Conclusion: Obesity prevalence has increased in IBD patients. This is significant because of the known increased levels of postoperative complications, perianal disease and requirement for more aggressive medical therapy. Research needs to be done to look at the effects of obesity on the co-mor- bid associations of other diseases with IBD, in particular colorectal cancer, and to ascertain whether or not screening frequency should be altered depending on BMI.

\section{Introduction}

Lower BMI has been associated with higher rates of inflammatory bowel disease (IBD) in men $(\mathrm{P}<0.01)$ [1]. Obesity is traditionally considered unusual in Crohn's disease (CD), but studies of obese patients have linked it to an increase in morbidity, with more frequent anoperineal complications and increased disease activity on an annual basis [2].

Obesity in Scotland is increasing: $64 \%$ of men and $57 \%$ of women were overweight in 2003, representing an increase from 1995 and a greater increase in the obese proportion of the population [3]. Previous studies of the juvenile IBD population diagnosed between 1963 and 1983 and followed up in adult life showed that the CD population had significantly lower body weight than people with ulcerative colitis (UC) and significantly lower BMI than the general population, whereas all UC patients had normal BMIs [4].

The aim of this retrospective study was to assess the distribution of body mass indices in the IBD population of Tayside, Scotland.

\section{Material and Methods}

The IBD population of Tayside comprises a total of 1,269 patients. To be included in the final analysis, the weight obtained from patients' notes had to be taken within the preceding 12 months. In order to avoid inaccurate BMI measurements, height measurements had to have occurred within the preceding decade. Patients over the age of 18 years and aged

\section{KARGER}

Fax +497614520714

Information@Karger.de

www.karger.com (c) 2009 S. Karger GmbH, Freiburg

Accessible online at:

www.karger.com/ofa
Dr. Helen Steed

Department of Gastroenterology, The Princess Royal Hospital

Telford, Shropshire,TF1 6TF, UK

Tel. +44 1952641222

helensteed@doctors.org.uk 
Table 1. BMI in IBD patients in Tayside

\begin{tabular}{|c|c|c|c|c|c|c|}
\hline \multirow[t]{2}{*}{ BMI, $\mathrm{kg} / \mathrm{m}^{2}$} & \multicolumn{2}{|c|}{ Patients with $\mathrm{CD}$} & \multicolumn{2}{|c|}{ Patients with UC } & \multirow[t]{2}{*}{ Total IBD, \% } & \multirow{2}{*}{$\begin{array}{l}\text { IBD in the general } \\
\text { Scottish population, \% }\end{array}$} \\
\hline & $\mathrm{n}$ & $\%$ & $\mathrm{n}$ & $\%$ & & \\
\hline$<18.5$ & 9 & 3 & 1 & 0.5 & 1 & 2 \\
\hline $18.5-24.9$ & 133 & 45 & 74 & 38 & 43 & 38 \\
\hline $25-29.9$ & 99 & 34 & 85 & 44 & 38 & 38 \\
\hline $30-34.9$ & 36 & 12 & 24 & 12 & 12 & 20 \\
\hline $35-39.9$ & 16 & 5 & 9 & 5 & 5 & $<2$ \\
\hline$>40$ & 2 & 1 & 1 & 0.5 & 1 & $<1$ \\
\hline
\end{tabular}

less than 28 , were required to have a height measurement performed during that time period in order to be included. The few patients in the adult service under the age of 18 had to have both a height and weight measurement in the preceding 12 months in order to be included into the study. All weights and heights were obtained by trained professionals on regularly standardised scales and a professionally fitted, wall-mounted height measure. Weight measurements were available for all patients, but height measurements were not. Patients were excluded if they were known to be body builders, pregnant or professional athletes or had undergone bariatric surgery. A total of 489 patients, 194 with UC and 295 with CD, were included in the final analysis ( $38.5 \%$ of the total IBD population). The 1995 WHO Obesity Classification was used to define obesity.

Statistics

Fischer's exact test was used to assess statistical significance.

\section{Comparison Population}

The Scottish population statistics obtained from the most recent national census were purchased from the General Register Office for Scotland and represent a comparison general population.

\section{Results}

Analysis revealed that $18 \%$ of the IBD population were obese, and there was very little difference in that percentage between $\mathrm{CD}$ and UC patients (table 1). Comparisons in all weight groups between the IBD population and the Scottish population were all non-significant.

In the obese group, defined as BMI $\geq 30.0 \mathrm{~kg} / \mathrm{m}^{2}, 19 \%$ had required surgery for their disease, compared to $18 \%$ of those being overweight, $46 \%$ of those of normal weight, and $10 \%$ of those who were underweight. These figures were strongly influenced by the high level of surgery for patients with CD (table 2).

Of the overweight UC patients, $60 \%$ were male and $40 \%$ female, compared to $48 \%$ male CD patients and $52 \%$ female, with the male to female ratio for both groups being not significantly different to that of the respective general population. In contrast, of the obese UC patients, $56 \%$ were male and $44 \%$ female, compared to $41 \%$ male and $59 \%$ female CD patients showing significant differences for both patient groups $(\mathrm{P}=$ $0.05,95 \%$ CI $0.46-0.66$ and $0.31-0.51$ ) when compared with the respective general population. Of the total overweight IBD patients, $54 \%$ were male and $46 \%$ female, and of the obese IBD patients $47 \%$ were male and $53 \%$ were female.
Table 2. Percentage of patients who have had surgery for their IBD

\begin{tabular}{llll}
\hline & UC patients & CD patients & Total \\
\hline Underweight & 0 & 11 & 10 \\
Normal & 5 & 34 & 46 \\
Overweight & 9 & 26 & 18 \\
Obese & 3 & 29 & 19 \\
\hline
\end{tabular}

\section{Discussion}

The results presented here showed a significant increase in the percentage of IBD patients with obesity. In our study, $18 \%$ of the $\mathrm{CD}$ population is obese, and a total of $52 \%$ is overweight or obese. In contrast, previous studies have reported $3 \%$ of the $\mathrm{CD}$ population as being obese and $32.4 \%$ of this population with a BMI of $25 \mathrm{~kg} / \mathrm{m}^{2}$ or higher [2]. Thus our data suggest a dramatic redistribution in the reported BMI of patients with IBD. However, this in line with the increase of obesity in the general population.

This increase is of clinical significance because overweight CD patients have been found to require surgery earlier in their disease course than their underweight counterparts [5], and obese patients who undergo ileoanal pouch formation have a higher risk of post-operative complications $(p=0.006)$, including wound infection and anastomotic separation [6]. It can be expected that this increase in the percentage of overweight or obese IBD patients result in higher numbers of surgical interventions, increased rates of post-operative complications, a need for earlier aggressive medical management, and consequently increased costs in management of IBD patients.

Obesity and UC may have inflammatory markers in common. In a case report on bariatric surgery in an obese UC patient [7], it could be shown that the patient's colitis, possibly due to a reduction in common inflammatory markers, improved after surgery. But further studies supporting this hypothesis are not available thus far. However, bariatric surgery has been shown to be potentially curative in other obesity-related conditions, such as diabetes. More research is necessary to elucidate whether or not bariatric surgery is a useful tool in the treatment of moderately or severely obese patients with active UC. 
The aetiology of IBD is multifactorial and still poorly understood. Dietary factors have been assessed as an aetiological factor, and, as described several decades ago, a diet higher in refined sugar and low in fibre, fruit and vegetables is accompanied by an increased incidence of IBD $[8,9]$. However, despite the dramatic increase in obesity in a western IBD population, widespread increases in the incidence of CD have not been reported. This suggests that the role of diet in IBD needs to be re-examined and assessed. Dietary factors need to be linked to gut microbiota, which has been identified as a potential aetiological factor in both IBD and, more recently, obesity. Such research may allow the development of new probiotic and synbiotic strategies for the management of these conditions both separately and in combination.

The increase of obesity in the general population is mirrored in the IBD subgroup. This result was unexpected as traditionally malnutrition and a reduced BMI have been cornerstones in the clinical assessment of IBD and also played a significant role in the gold standard for CD assessment, the Crohn's Disease Activity Index [10]. However, when considering the data presented here demonstrating widespread obesity amongst the IBD population, BMI itself can no longer be considered an accurate parameter for IBD assessment, and focus must shift away from it as it may lead to an underestimation of disease activity and an unwitting exclusion of obese IBD patients from clinical trials.

Currently there is no evidence that obesity in CD significantly alters the long-term course of the disease [2, 5]. IBD is also associated with increased rates of other diseases such as ischaemic heart disease, respiratory disorders, liver disorders, strokes, and diabetes. Obesity is also independently associated with a number of diseases that that have been shown to have also associations with IBD $[11,12]$. IBD patients are screened for colon cancer because of the increased risk [11], and cross-sectional studies have linked increased BMI to an increased prevalence of adenomas [13, 14]. As yet there are no studies dealing with co-morbid disease incidence in the obese IBD population. With obesity prevalence in the IBD population reflecting that of the general population and with those levels expected to rise, further research into this aspect of IBD care is warranted, with a particular focus on colorectal cancer. It may be that screening frequency for colorectal cancer could be increased or decreased, depending on disease and BMI.

In conclusion, obesity has become commonplace in IBD. But, in epidemiological terms, little is known about the potentially large-scale impacts this may have on management, cost, complications and co-morbid associations. The needs of these patients have been demonstrated to be slightly different to those of the non-obese IBD patients. Clinical and epidemiological research is required to better define obesity as a process within IBD and to highlight the differences, in order to understand whether or not a subtle shift in the treatment approaches and in the timing of currently available management strategies is required.

\section{Disclosure}

The authors declared no conflict of interests.

\section{References}

1 Landau DA, Goldberg A, Levi Z, Levy Y, Niv Y, Bar-Dayan Y: The prevalence of gastrointestinal disease in Israeli adolescents and its association with body mass index, gender and Jewish ethnicity. J Clin Gastroenterol 2008;42:903-909.

2 Blain A, Cattan S, Beaugerie L, Carbonnel F, Gendre JP, Cosnes J: Crohn's disease clinical course and severity in obese patients. Clin Nutr 2002;21: 51-57.

3 The Scottish Government: Health of Scotland's Population - Healthy Weight. August 27, 2008.

4 Ferguson A, Sedgwick DM: Juvenile onset inflammatory bowel disease: height and body mass index in adult life. BMJ 1994;308:1259-1263.

5 Hass DJ, Brensinger CM, Lewis JD, Lichtenstein GR: The impact of increased body mass index on the clinical course of Crohn's disease. Clin Gastroenterol Hepatol 2006;4:482-488.
6 Kiran RP, Remzi FH, Fazio VW, Lavery IC, Church JM, Strong SA, Hull TL: Complications and functional results after ileoanal pouch formation in obese patients. J Gastrointestinal Surg 2008; 12:668-674.

7 Lascan CA, Soto F, Carrodeguas L, Szomstein S, Rosenthal RJ, Wexner SD: Management of ulcerative colitis in the morbidly obese patient: is bariatric surgery indicated? Obes Surg 2006;16:783-786.

8 Gilat T, Hacohen D, Lilos P, Langman MJS: Childhood factors in ulcerative colitis and Crohn's disease. An international cooperative study. Scand J Gastroenterol 1987;22:1009-1024.

9 Thornton JR, Emmett PM, Heaton KW: Diet and Crohn's disease: characteristics of the pre-illness diet. BMJ 1979;ii:762-764.

10 Best WR, Becktel JM, Singleton JW, Kern F Jr: Development of a Crohn's Disease Activity Index. Gastroenterology 1976;70:439-444.
11 Garrow JS: Obesity and Related Diseases. Edinburgh, Churchill Livingstone, 1988.

12 Willet WC, Green A, Stampfer MJ, Speizer FE, Colditz GA, Rosner B, Monson RR, Stason W, Hennekens $\mathrm{CH}$ : Relative and absolute excess risks of coronary heart disease among women who smoke cigarettes. N Engl J Med 1987;317:1303-1309.

13 Munkholm P, Langholz E, Davidsen M, Binder V: Intestinal cancer risk and mortality in patients with Crohn's disease. Gastroenterology1993;105:17161723.

14 Yamaji Y, Okamoto M, Yoshida H, Kawabe T, Wada R, Mitsushima T, Omata M: The effect of body weight reduction on the incidence of colorectal adenoma. Am J Gastroenterol 2008;103:20612067. 